International Journal of Engineering \& Technology, $10(2)(2021) 164-169$
International Journal of Engineering \& Technology
SPC
Website: www.sciencepubco.com/index.php/IJET
Research paper

\title{
Numerical simulation of the influence of pores sizes on moisture migration dynamic in masonry wall
}

\author{
N. M. Nde ${ }^{1 *}$, D. Fokwa ${ }^{1}$, M. Mbessa ${ }^{2}$, T. T. Tamo ${ }^{3}$, C. Pettang ${ }^{4}$ \\ ${ }^{1}$ Department of Civil Engineering, university of Douala, Douala-Cameroon \\ ${ }^{2}$ Department of Civil Engineering, university of Yaounde, Yaounde-Cameroon \\ ${ }^{3}$ Department of Civil Engineering, university of Dschang, Dschang-Cameroon \\ ${ }^{4}$ Department of Civil Engineering, university of Bamenda, Bamenda-Cameroon \\ *Corresponding author E-mail: martialnde@yahoo.fr
}

\begin{abstract}
The sometimes extreme hydro-climatic stresses that buildings undergo can lead to significant deterioration which can lead to their collapse. The concern to realize durable works and ensuring a comfortable framework for the life of occupants leads to seek effective solutions, as well for the new construction as for the renovation of old construction, answering the sempiternal problem of harmful action of water on buildings materials. This paper proposes a numerical simulation of moisture migration in concrete building walls, the aim being to highlight the influence of pore size on the kinetics of moisture migration, and its gradient in the wall. A mathematical model taking into account the mechanisms of moisture migration due to liquid moisture gradient and by vapor diffusion is proposed; the discrete formulation of the equation by the numerical scheme of Crank Nicolson is then carried out, and results from computer modeling using Matlab software version 7.10.0.499 (R2010a), show that pore size is a key parameter that influences the dynamics of moisture migration in the wall. Indeed, this parameter qualitatively and quantitatively influences the kinetics of moisture migration, as well as it gradient in the concrete wall. It appears a greater migration dynamic when the pores sizes decrease, means a greater kinetics of moisture migration and lower moisture gradient in the walls at the hygrometric equilibrium, for a decreasing pore size.
\end{abstract}

Keywords: Mathematical Modeling; Migration; Moisture; Numerical Simulation; Pore Size

\section{Introduction}

Construction materials are mostly parous media, and therefore subject to almost permanent moisture exchange with the environment around them. Among these construction materials, concrete is the most widely used in the world, and its water content due to its porous character, is an important parameter in terms durability of concrete structures. The influence of this porosity on the dynamics of moisture migration in concrete has been the subject of several publications. Thus, several authors have conducted research on the dynamics of water migration in concrete, going as far as modifying the porous structure of concrete thanks to various additives, in order to highlight the influence of these additives on the kinetics of migration and the moisture gradient in concrete. Ivan Lukic [1] shows that mineral additives increase the porosity of concrete, leading to high capillary absorption; Nilforoushan, and Reza [2] show that micro silica modifies pore size distribution and permeability of type $\mathrm{V}$ cement concrete; in fact, they show that the permeability of concrete with addition of micro silica decrease due to modification in pore size distribution. Similarly, Zineb Bajja [3] studied the microstructure of paste, mortars and concrete based on cement (CEM I), with silica addition, and shows that this addition modifies the microstructure and then influences the moisture transport properties. Moussa and others [4] show that the replacement of cement by iron powder improve the porosity and influence the water absorption of hardened concrete, specially the water absorption of the hardened concrete increase when the percent content of iron powder increase. Further on, Oltulu and Sahin [5], Mohseni and others [6] highlight the combined effects of nano-particles: nano- $S_{i} O_{2}$, nano$\mathrm{Al}_{2} \mathrm{O}_{3}$, nano- $\mathrm{Fe}_{2} \mathrm{O}_{3}$, nano- $\mathrm{TiO}_{2}$ powder on the water absorption dynamic. Djima and others [7] show the increasing of water absorption of concrete as the lime treated palm kernel shell and sugarcane bogasse ash increase in the concrete mixture. Malab and others [8] made comparative study of the drying kinetics of self-compacting concrete with those of an ordinary concrete and sand concrete, and show the first one with macropores has lower drying kinetics than the second with mesopores; these conclusions are also confirmed by Goual and others results [9], who show that the presence of macropores considerably attenuates moisture transport of civil engineering materials in general. Similarly, according to Suchorab and others [10], laboratory experiment and computer modeling confirm strong capillary properties of aerated concrete. Some authors have carried out research on the composition parameters of concrete and their influence on the porous structure and the moisture transport properties in concrete; thus Ghashghali and Hassani [11] show that characteristics such as water permeability and porosity present a clear dependence on the size of aggregate and mix design parameters; in fact the porosity and consequently water permeability coefficient decrease when the constant water to cement ratio (W/C) of pervious concrete increase. Suchorab [12] shows that the impregnation of building materials in hydrophobic solutions modifies their porosity leading to decreasing in dynamics 
of capillary moisture migration. However, even if these works take into account the porosity of materials, they do not explicitly highlight the pore size influence in the moisture migration; in other words, they studies carried out are sometimes contradictory and do not clearly present the role of pore size on the moisture migration dynamic in porous material. This work is part of the overall objective of highlighting the influence of pore sizes on the moisture migration dynamics. Specifically to show the influence of pore size on migration kinetics and moisture gradient in the wall. So, this paper first presents a mathematical modeling of moisture migration in a single layer wall; then, the results of numerical simulation are analyzed.

\section{Material and method}

To study the water migration in based of buildings, a mathematical model is proposed and then the numerical simulations are done with Matlab software. The method is based on finite differences and consists in defining an optimal geometry, space and time discretization, initial and boundary conditions.

\subsection{Mathematical modelling}

The model is based on the Philip and De Vries equations; moisture moves in the liquid and vapor state, with the moisture potential as the common potential. In this approach, the scale is assumed to be macroscopic, where the parous medium is considered to be an equivalent fictitious continuous medium; transfers are unsteady and one-dimensional; the different states (liquid and vapor) are in thermal equilibrium at any point in the parous medium; the capillary pores are assumed to be parallel and cylindrical. The liquid and vapor moisture flows are given according to Philip and De Vries [13] respectively by Equation (1) and Equation (2) while neglecting the gravity flow:

$$
\begin{aligned}
& \mathrm{J}_{1}=-\mathrm{D}_{1} \overrightarrow{\operatorname{grad}}(m)-\mathrm{D}_{\text {Iт }} \overrightarrow{\operatorname{grad}}(\mathrm{T}) \\
& \mathrm{J}_{\mathrm{v}}=-\mathrm{D}_{\mathrm{v}} \overrightarrow{\operatorname{grad}}(m)-\mathrm{D}_{\mathrm{v}} \overrightarrow{\operatorname{grad}}(m)
\end{aligned}
$$

$J_{l}$ and $J_{v}$ represent the liquid and vapor moisture flow respectively, $D_{l}$ and $D_{v}$ respectively the liquid and vapor moisture diffusion coefficients. $D_{l T}$ and $D_{v T}$ respectively the liquid and vapor moisture diffusion coefficients due to temperature gradient. T the temperature and m moisture content (humidity).

At the liquid-gas thermodynamic equilibrium, Thomson's equation [14] gives the relationship between relative humidity, temperature and pore radius as presented in Equation (3), and transformed to get Equation (4):

$$
\begin{aligned}
& \mathrm{P}_{\mathrm{v}} / \mathrm{P}_{0 \mathrm{v}}=\mathrm{m}=\operatorname{esp}\left[\left(-2 \times \mathrm{T}_{\mathrm{s}} \times \gamma_{\mathrm{w}} \cos \beta\right) /(\mathrm{r} \times \mathrm{R} \times \mathrm{T})\right] \\
& \mathrm{T}=\left(-2 \times \mathrm{T}_{\mathrm{s}} \times \lambda_{\mathrm{w}} \times \cos \beta\right) /(\mathrm{r} \times \mathrm{R} \times \operatorname{lnm})
\end{aligned}
$$

$P_{v}$ and $P_{0 v}$ are respectively the vapor pressure and the saturated vapor pressure; $T_{s}$ the surface tension; $\gamma_{W}$ the molar volume of water; $\mathrm{r}$ the pore radius; $\mathrm{R}$ the perfect gas constant and $\beta$ the water contact angle.

A coupling of Equation (1), Equation (2), and Equation (4) lead to Equation (5):

$$
(\partial m / \partial \mathrm{t})=\mathrm{F}_{1}(m) \times\left(\partial^{2} \mathrm{~m} / \partial \mathrm{x}^{2}\right)+\mathrm{F}_{2}(m) \times(\partial m / \partial \mathrm{x})^{2}
$$

With $F_{1}(m)$ and $F_{2}(m)$ the moisture migration coefficients, function of the moisture $m$ and given by the Equation (6) and Equation (7):

$$
\begin{aligned}
& \mathrm{F}_{1}(\mathrm{~m})=\mathrm{D}_{\mathrm{w}}-\left(\mathrm{D}_{\mathrm{T}} / \mathrm{T}_{0}\right) \times\left(\mathrm{a} / \mathrm{m} \times(\mathrm{lnm})^{2}\right) \\
& \mathrm{F}_{2}(\mathrm{~m})=\left(\mathrm{D}_{\mathrm{T}} / \mathrm{T}_{0}\right) \times\left[(\mathrm{a} \times(2+\mathrm{lnm})) /\left(\mathrm{m}^{2} \times(\operatorname{lnm})^{3}\right)\right]
\end{aligned}
$$

Where a is a coefficient defined to simplify writing. $D_{W}$ the moisture diffusion coefficients due to water content gradient. And $D_{T}$ the moisture diffusion coefficients due to temperature gradient [13].

$$
\begin{aligned}
& \mathrm{a}=\left(-2 \times \mathrm{T}_{\mathrm{s}} \times \gamma_{\mathrm{w}} \times \cos \beta\right) /(\mathrm{r} \times \mathrm{R}) \\
& \mathrm{D}_{\mathrm{w}}=\mathrm{D}_{1}+\mathrm{D}_{\mathrm{v}} \\
& \mathrm{D}_{\mathrm{T}}=\mathrm{D}_{\mathrm{IT}}+\mathrm{D}_{\mathrm{vT}}
\end{aligned}
$$

\subsection{Linearization}

The computational of the homogeneous and nonlinear Equation (5) by the finite difference method requires to linearize it. An approximation is therefore made on the coefficients $F_{1}(m)$ and $F_{2}(m)$, they are discrete and supposed known at time $\mathrm{j}$ and abscissa $\mathrm{i}$. we then obtain Equation (11) and Equation (12): 
$F_{1}\left(m_{i}^{j}\right)=D_{w}-\left(D_{T} / T_{0}\right) \times\left[a /\left(m_{i}^{j} \times\left(\operatorname{lnm} m_{i}^{j}\right)^{2}\right)\right]$

$\mathrm{F}_{2}\left(\mathrm{~m}_{\mathrm{i}}^{\mathrm{j}}\right)=\left(\mathrm{D}_{\mathrm{T}} / \mathrm{T}_{0}\right) \times\left[\mathrm{a} \times\left(\operatorname{lnm}_{\mathrm{i}}^{\mathrm{j}}+2\right) /\left(\left(\mathrm{m}_{\mathrm{i}}^{\mathrm{j}}\right)^{2}\left(\operatorname{lnm}_{\mathrm{i}}^{\mathrm{j}}\right)^{3}\right)\right]$

\subsection{Geometry and discretization}

The diagram in Figure 1 illustrates the spatial and temporal discretization of the studied geometry.

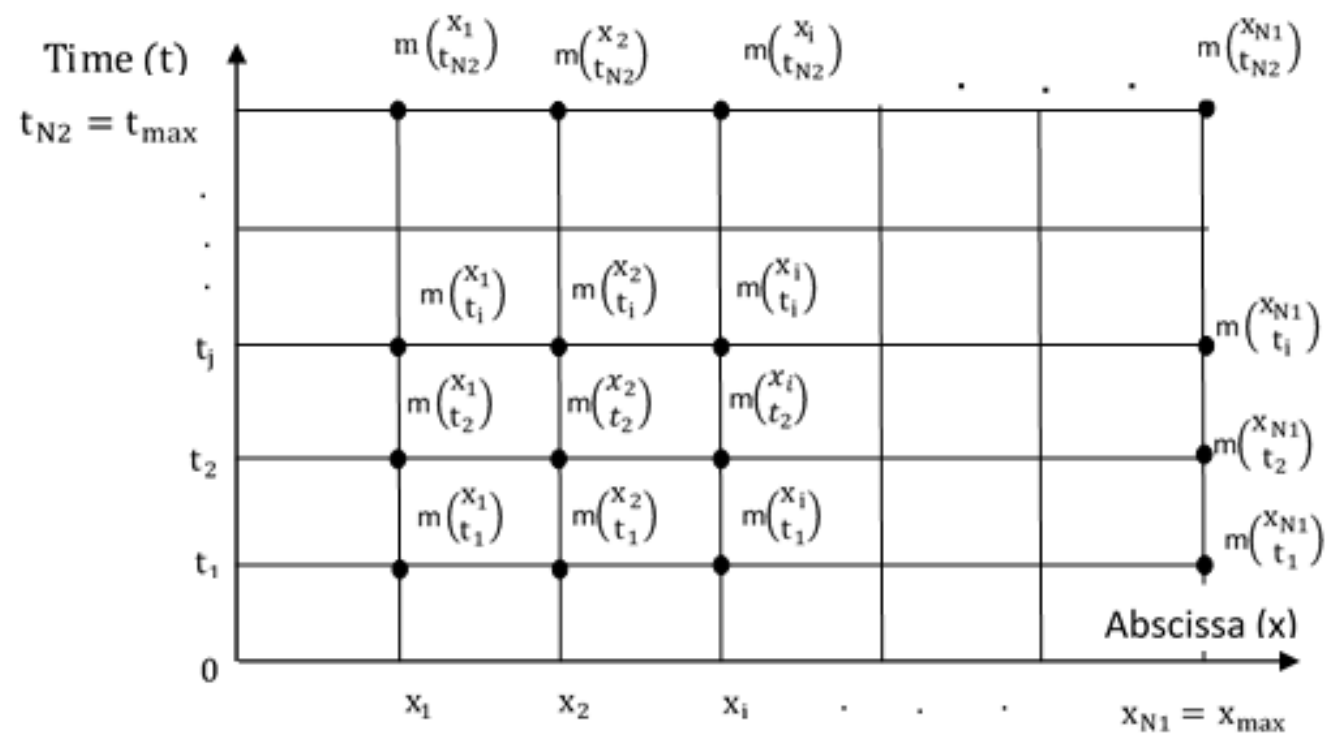

Fig. 1: Spatial and Temporal Discretization.

The progressive finite differences method is used to discretize moisture flow, given by Equation (13); and the centered finite differences method discretize the moisture gradient, given by Equation (14)

$\partial \mathrm{m}(\mathrm{x}, \mathrm{t}) / \partial \mathrm{t}=\left(\mathrm{m}_{\mathrm{i}}^{\mathrm{j}+1}-\mathrm{m}_{\mathrm{i}}^{\mathrm{j}}\right) / \mathrm{T}_{2}$

$\partial^{2} \mathrm{~m}(\mathrm{x}, \mathrm{t}) / \partial \mathrm{t}=\left(1 / \mathrm{h}^{2}\right) \times\left(\mathrm{m}_{\mathrm{i}+1}^{\mathrm{j}}-2 \mathrm{~m}_{\mathrm{i}}^{\mathrm{j}}+\mathrm{m}_{\mathrm{i}-1}^{\mathrm{j}}\right)$

With $T_{2}$ and $\mathrm{h}$ respectively the temporal and spatial discretization rates, defined by Equations (15) and Equation (16):

$$
\begin{aligned}
& \mathrm{T}_{2}=\mathrm{t}_{\text {max }} / \mathrm{N}_{2} \\
& \mathrm{~h}=\mathrm{e} / \mathrm{N}_{1}
\end{aligned}
$$

e is the wall thickness, $t_{\max }$ the duration of moisture migration, $N_{1}$ and $N_{2}$ respectively the number of space and time steps.

\subsection{Discrete formulation of the equation by crank Nicolson scheme}

For the discrete formulation of Equation (5), the second coefficient $F_{2}(m)$ is excluded because its influence on the migration dynamics is negligible. Thus, the discrete scheme of Crank Nicolson, of Equation (17) leads to the algebraic equation system, Equation (18):

$(\partial \mathrm{m} / \partial \mathrm{t})=\mathrm{F}_{1}(\mathrm{~m}) \times\left(\partial^{2} \mathrm{~m} / \partial \mathrm{x}^{2}\right)$

$\left[\mathrm{M}_{2}\right]\left\{\mathrm{m}^{\mathrm{j}+1}\right\}+\left\{\mathrm{N}_{\mathrm{ak}}\right\}=\left[\mathrm{M}_{1}\right]\left\{\mathrm{m}^{\mathrm{j}}\right\}+\left\{\mathrm{N}_{\mathrm{al}}\right\}$

$\left[M_{1}\right]$ and $\left[M_{2}\right]$ are the material characteristics matrix. The matrix of boundaries conditions are given at time $\mathrm{j}$ and $\mathrm{j}+1$ respectively by $\left\{N_{a l}\right\}$ and $\left\{N_{a k}\right\}$.

\subsection{Initial and boundaries conditions, calculation parameters}

The initial conditions and the boundaries conditions in moisture, applied to the geometry are defined as follows:

For $\mathrm{x}=0, \mathrm{~m}(0, \mathrm{t})=\mathrm{m}_{0}(\mathrm{t}) \forall \mathrm{t} \geq 0$ 
For $\mathrm{x}=\infty, \mathrm{m}(\infty, \mathrm{t})=\mathrm{m}_{0}(\mathrm{t}) \forall \mathrm{t} \geq 0$

$$
\text { For } \mathrm{t}=0, \mathrm{~m}(\mathrm{x}, 0)=\mathrm{m}_{\mathrm{i}}(\mathrm{t}=0) \forall \mathrm{x}
$$

The numerical values of the parameters used in this simulation come from Bordachev's work on moisture calculation analysis and injection methods in brick masonry walls, where he shows that the revetment based on a material with high porosity or its injection into the wall basement, considerably limits the moisture migration in the wall [15]. Furthermore, some numerical values of parameters were taken from Kiwan's work, on the reliability of energy performance in buildings [16]. Simulations are performed using Matlab software (version 7.10.0.499 (R2010a)), varying the pore size.

\section{Results and discussions}

To highlight the influence of the pore size on moisture migration dynamic in porous wall, the map of Figure 2 show the spatial and temporal moisture distribution in the wall; while graphs of Figure 3 and Figure 4 to Figure 6 respectively present the spatial and temporal evolution curves of this moisture, for different pore sizes. These curves are qualitatively in agreement with Abahri et al's contribution to the analytical and numerical study of combine heat and moisture transfer in parous building materials [17]; Fitsum et al's work on the transient model for coupled heat, air and moisture transfer through multilayered parous media [18]; Ketelaar et al's results on the comparison of diffusion coefficients from moisture concentration profile and drying curve [19]; and Nytsch-Geusenet et al's work on the object oriented language 'Modelica' for the monitoring of heat and moisture in the building [20]. The observed deviations can be justified by the boundary conditions and the moisture diffusion coefficient of the materials.

\subsection{Moisture mapping in the geometry}
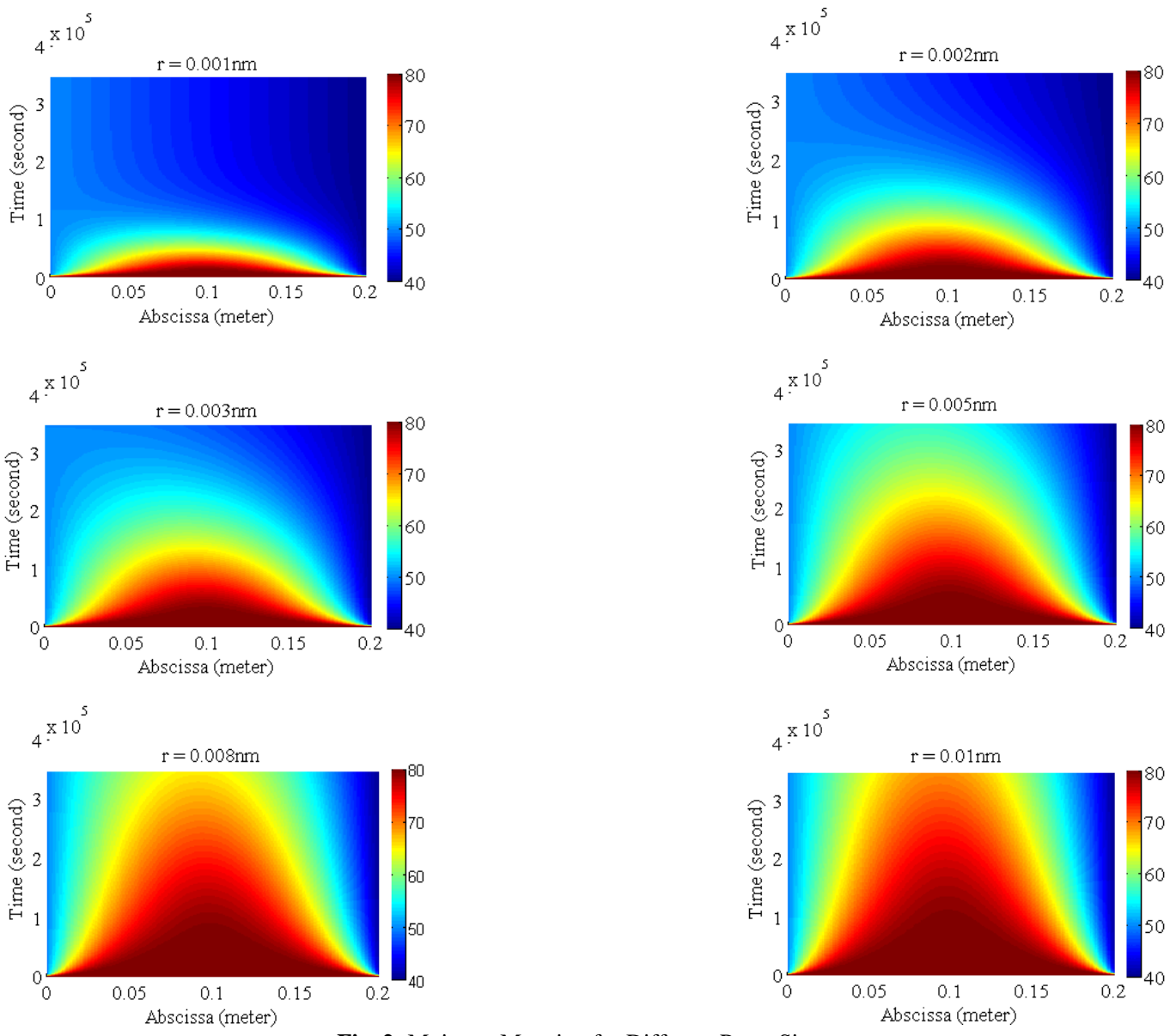

Fig. 2: Moisture Mapping for Different Pores Sizes.

The maps of Figure 2 show on the one hand a low diffusivity in the center of the wall, this independently of pores sizes; and on the other hand a moisture concentration attenuated over time, for smaller pores radius. This can be explained by the fact that the capillary pressure which leads to the moisture migration in the wall increases as the capillary radius decreases. Thus, at the end of the migration (final state), at hygrometric equilibrium the moisture concentration gradient in the wall is less important; and the migration kinetics leading to this equilibrium is increasing when the pore size decreases.

\subsection{Spatial evolution of moisture}

The curves in Figure 3 illustrate for each time of simulation, the evolution of moisture content in the wall made of two different pores radius. The red curves correspond to the largest pore radius $0.003 \mathrm{~nm}$, while the blue curves correspond to the smallest pore radius $0.001 \mathrm{~nm}$. 
It appears a faster migration for the smallest pore radius; indeed, for this pore size, the moisture balance is reached after 345600 s, against 518400s for largest pore radius. This result explicitly highlights the influence of pore size on the spatial evolution of moisture in the wall.

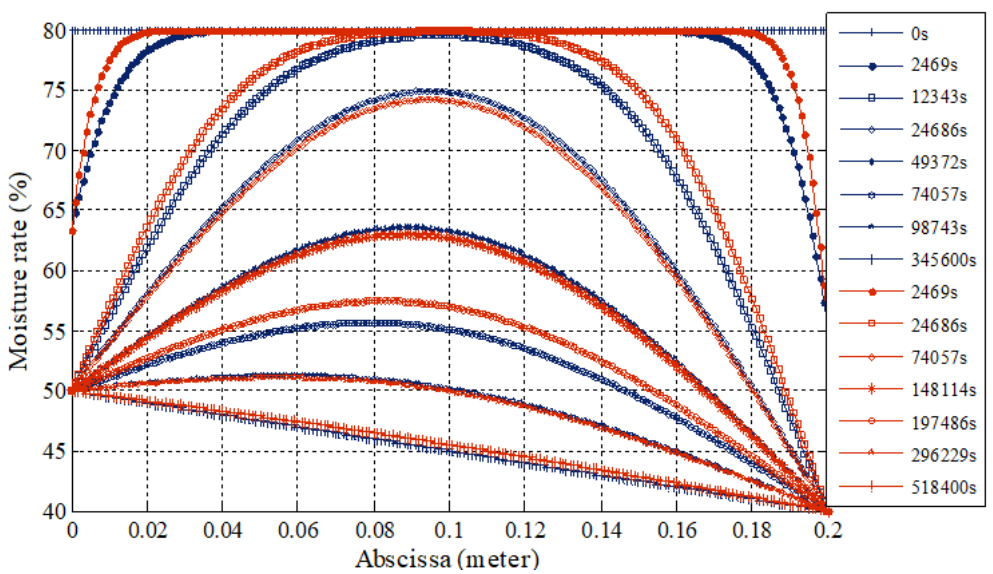

Fig. 3: Spatial Evolution of Moisture at Different Times of Simulation, for Different Pores Sizes: 0.003nm (Red Curves), 0.001nm (Blue Curves).

\subsection{Temporal evolution of moisture}

The result of Figure 4 presents the temporal evolution of moisture at different abscissa of the wall, for a pore radius $0.003 \mathrm{~nm}$. The general shape of the curves is the same for each variation on each abscissa; the curves decrease more or less quickly, then evolve in level (almost stationary evolution in time). Furthermore the slopes of these curves are increasingly steep, for abscissa which move away from the middle of the wall $(\mathrm{x}=0.10 \mathrm{~m})$, and the plateau is quickly reached for the abscissas located near from the edges of the wall. Indeed, the slopes of these curves materializes the kinetics with which the material reaches its hygrometric equilibrium at a given abscissa in the wall. The diffusion is therefore faster at the edges of the wall than towards the inside thereof. This can be explained by the fact that the edges of the wall during the simulation time are influenced by the boundary conditions; their hygrometric balance is thus quickly reached compared to that of the middle of the wall.

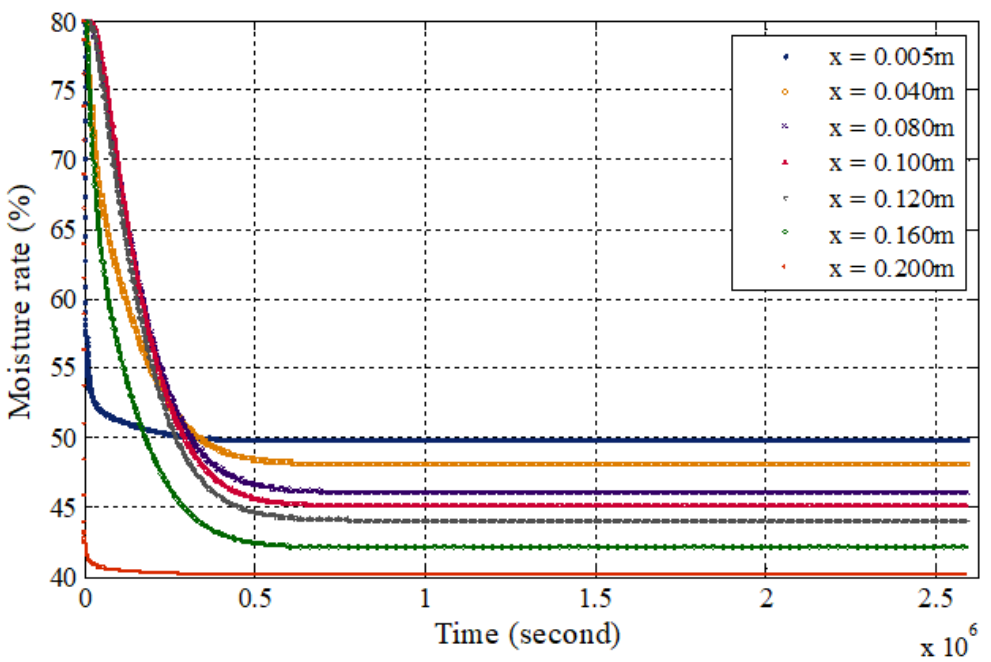

Fig. 4: Temporal Evolution of Moisture at Different Abscissa of the Wall for A Pore Radius of 0.001nm.

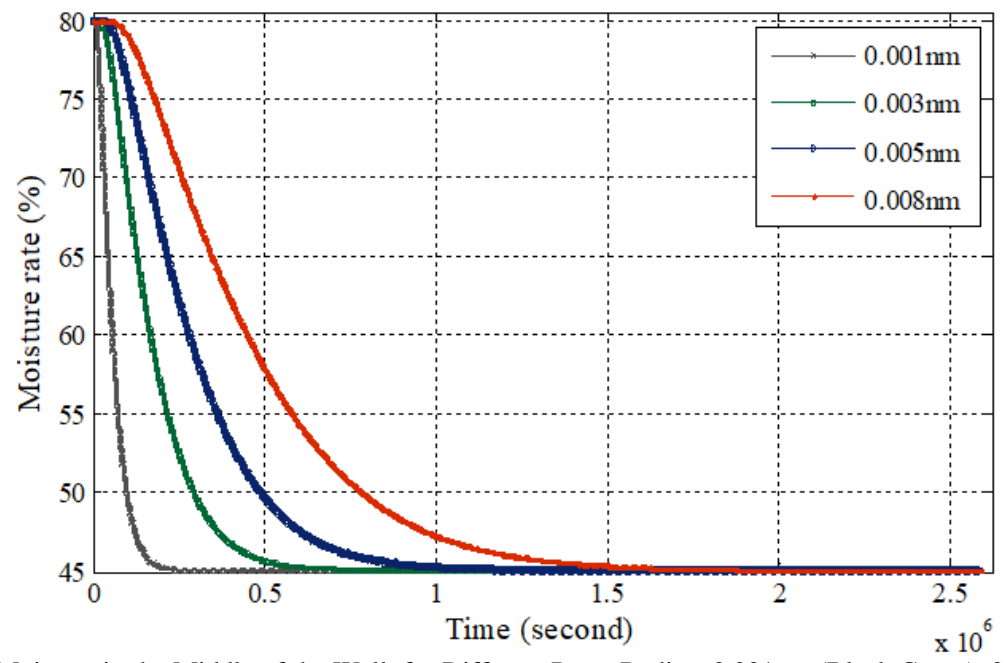

Fig. 5: Temporal Evolution of Moisture in the Middle of the Wall, for Different Pores Radius, 0.001nm (Black Curve), 0.003nm (Green Curve), 0.005nm (Blue Curve) and 0.008nm (Red Curve) 
Figure 5 above presents the temporal evolution of the moisture in the middle of the wall, for different pores sizes: $0.001 \mathrm{~nm}$ (black curve), $0.003 \mathrm{~nm}$ (green curve), $0.005 \mathrm{~nm}$ (blue curve) and $0.008 \mathrm{~nm}$ (red curve). The curves have the same decreasing appearance then evolve in level, reflecting a state of hygrometric equilibrium in the material. However, this state of equilibrium is quickly reached for the wall with the smallest pore size. Indeed around a duration of $250000 \mathrm{~s}(72 \mathrm{~h})$, this hygrometric balance is reached in the wall of porosity $0.001 \mathrm{~nm}$; against a duration of $518400 \mathrm{~s}(144 \mathrm{~h})$ in the wall of pore size $0.003 \mathrm{~nm}$, a duration greater than $1875000 \mathrm{~s}(521 \mathrm{~h})$ in the wall of pore size $0.005 \mathrm{~nm}$, and a duration greater than $2400000 \mathrm{~s}(667 \mathrm{~h})$ in the wall of pore size $0.008 \mathrm{~nm}$.

The curves in Figure 6 illustrate the evolution over time, of the moisture in the wall, for different abscissas in the wall corresponding to two pores sizes. The curves in solid lines correspond to the largest pore size $0.003 \mathrm{~nm}$, while the curves in dashed lines correspond to the smallest pore size $0.001 \mathrm{~nm}$. These curves are qualitatively identical, decreasing in appearance and then evolving in level, reflecting a state of hygrometric equilibrium in the wall. However, regardless of the abscissa $\mathrm{x}$, this equilibrium is reached quickly for the wall whose pore size is the smallest.

\section{Conclusion}

This paper presents a numerical simulation of moisture migration in concrete building walls, the influence of pore size on the dynamic of moisture migration has been highlighted, and it appears a low diffusivity in the center of the wall, this independently of pores sizes. Furthermore, a greater migration dynamic when the pores sizes decrease, means a greater kinetics of moisture migration and lower moisture gradient in the walls at the hygrometric equilibrium, for a decreasing pore size. These results find their applications in the choice of building materials their quality, their manufacture and their use for a better durability.

\section{References}

[1] Lukic, I. (2015) Influence of mineral admixtures on water absorption of lightweight aggregate concrete. Scientific Conference, Planing, Design, Construction and Building Renewal, NOVI SAD, 25-27.

[2] Nilforoushan, Reza, M. (2005) The effect of micro silica on permeability and chemical durability of concrete used in the corrosive environment. Iran. J. Chem. And Chem. Eng.

[3] Baija, Z. (2017) Influence de la microstructure sur le transport diffusif des pâtes, mortiers et béton à base de CEM I avec ajout de fumée de silice. Thèse de Doctorat, Université Paris -Saclay, Ecole normale Supérieure CACHAN, p. 245

[4] Moussa, A. L., Mutuku, R., and Thuo, Joseph. (2018) effect of iron powder $\left(\mathrm{Fe}_{2} \mathrm{O}_{3}\right)$ on strength workability, and porosity of the binary blended concrete. Open Journal of Civil Engineering, Scientific Research and Publishing, 8, 411-425.

[5] Oltulu, M. and Sahin, R. (2013) Effect of nano- $\mathrm{SiO}_{2}$, nano- $\mathrm{Al}_{2} \mathrm{O}_{3}$ and nano- $\mathrm{Fe}_{2} \mathrm{O}_{3}$ powder on compressive strength and capillary water absorption of cement mortar containing fly ash: comparative study. Energy and Building, 58, 292-301. https://doi.org/10.1016/j.enbuild.2012.12.014.

[6] Mohseni, E. et al. (2015) Single and combined effects of nano- $\mathrm{SiO}_{2}$, nano- $\mathrm{Al}_{2} \mathrm{O}_{3}$ and nano- $\mathrm{TiO}_{2}$ on the mechanical, rheological and durability properties of self-compacting mortar containing fly ash.

[7] Djima, M. O. A., Mang'uriu, N. G and Mwero, J. N. (2018) Experimental investigation of lime treated palm kernel shell and sugarcane bagasse ash as partial replacement of coarse aggregate and cement respectively in concrete. Open Journal of Civil Engineering, Scientific Research and Publishing, 8, 358-372. https://doi.org/10.4236/ojce.2018.84027.

[8] Malab, S., Benaissa, A., Boudraa, S. E and Aggoum, S. (2009) Drying kinetics of self-compacting concrete. Turkish Journal of Engineering and Environmental Science, 33, 135-145.

[9] Goual, M. S., Bali, A and Quéneudec, M. (2003) Influence de la structure poreuse sur le transfert d'humidité dans les matériaux poreux de génie civil "application au béton argileux cellulaire". Seminaire International de Géomatériaux GEOMAT’02, Université Mohamed Boudiaf de M'sila.

[10] Suchorab, Z. et al. (2014) Volatil organic compound protection against moisture in building materials. Ecological Chemistry and Engineering, p 12.

[11] Ghashghali, H. T. and Hassani, A. (2016) Investigating the relationship between porosity and permeability coefficient for pervious concrete pavement by statistical modelling. Material Sciences and Applications, Scientific Research and Publishing, 7, 101-107. https://doi.org/10.4236/msa.2016.72010

[12] Suchorab, Z., Widomski, M., Lagod, G and Sobczuk, H. (2010) Capillary rise phenomenon in aerated concrete. Monotoring and simulation. Lublin University of Technology.

[13] Philip, J.R. and De Vries, D.A. (1957) Moisture movement in porous materials under temperature gradients. s.l. : Transaction of American Geophysical Union, Vol. 38, pp. 222-232. https://doi.org/10.1029/TR038i002p00222.

[14] Thomson, W. (1871) On the equilibrium of vapour at a curved surface of liquid, Philosophical Magazine, series 4, 42(282), 448-452. https://doi.org/10.1080/14786447108640606.

[15] Bordachev, A. (2010) Moisture Calculation analysis and injection methods in brick masonry walls. Saimaa University of Applied Sciences, Lappeenranta, 71p.

[16] Lamis, K. (2012) Fiabilité Des Performances Energétiques Des Bâtiments. Mémoire de Master STEU, Université Libanaise, Faculté De Génie III Hadath.

[17] Abahri, K., Belarbi, R and Trabelsi, A. (2011) Contribution to analytical and numerical study of combined heat and moisture transfers in porous building materials. Building and Environment, 46, 1354 - 1360. https://doi.org/10.1016/j.buildenv.2010.12.020.

[18] Fitsum, T., Kumar, K and Fazio, P. (2010) Transient model for coupled heat, air and moisture transfer through multilayered porous media. International Journal of Heat and Mass Transfer, 53, 3035-3044. https://doi.org/10.1016/j.ijheatmasstransfer.2010.03.024.

[19] Ketelaars, A. A. J., Pel, L., Coumans, W. J and Kerkhof, P. J. (1995) Drying kinetics: a comparison of diffusion coefficients from moisture concentration profiles and drying curves. Chemical Engineering Science, 50, 1187-1191. https://doi.org/10.1016/0009-2509(94)00494-C.

[20] Nytsch-Geusenet et al. (2005) A hygrothermal building model based on the object-oriented modeling language modelica. Ninth International IBPSA Conférence, Montréal, Canada, 15-18. 